\title{
Curcumin Reduces the Noise-Exposed Cochlear Fibroblasts Apoptosis
}

\author{
Tengku Siti Hajar Haryuna ${ }^{1}$ Wibi Riawan ${ }^{2}$ Ardyansyah Nasution ${ }^{1} \quad$ Suprapto Ma'at $^{3} \quad$ Juliandi Harahap $^{4}$ \\ Indri Adriztina ${ }^{1}$
}

${ }^{1}$ Department of Otorhinolaryngology-Head and Neck Surgery, Faculty of Medicine, Universitas Sumatera Utara, Medan 20155, Indonesia

2 Department of Biochemistry, Faculty of Medicine, Universitas

Brawijaya, Malang 65145, Indonesia

${ }^{3}$ Department of Clinical Pathology, Faculty of Medicine, Universitas

Airlangga, Surabaya 60131, Indonesia

${ }^{4}$ Department of Community Medicine, Faculty of Medicine,

Universitas Sumatera Utara, Medan 20155, Indonesia

Int Arch Otorhinolaryngol 2016;20:370-376.
Address for correspondence Tengku Siti Hajar Haryuna, MD, ENT Specialist, PhD, Department of Otorhinolaryngology - Head and Neck Surgery, Faculty of Medicine, Universitas Sumatera Utara, Jalan Bunga Lau No. 17, Medan Tuntungan Medan, Sumatera Utara 20136, Indonesia (e-mail: tengkusitihajarharyuna@gmail.com).

\begin{abstract}
Introduction The structural changes underlying permanent noise-induced hearing loss (NIHL) include loss of the sensory hair cells, damage to their stereocilia, and supporting tissues within the cochlear lateral wall.

Objective The objective of this study is to demonstrate curcumin as a safe and effective therapeutic agent in the prevention and treatment for fibroblasts damage within the cochlear supporting tissues and lateral wall through cell death pathway.

Methods We divided 24 Rattus norvegicus into 4 groups, Group 1: control; Group 2: noise (+); Group 3: noise (+), 50 mg/day curcumin (+); Group 4: noise (+), $100 \mathrm{mg} /$ day curcumin (+). We provided the noise exposure dose at $100 \mathrm{~dB}$ SPL for two hours over two weeks and administered the curcumin orally over two weeks. We examined all samples for the expressions of calcineurin, nuclear factor of activated T-cells cytoplasmic 1 (NFATc1), and apoptotic index of cochlear fibroblasts.

Results We found significant differences for the expressions of calcineurin $(p<0.05)$

Keywords

- noise

- curcumin

- calcineurin

- NFATC1

- apoptosis

- cochlea in all groups, significant differences for the expressions of NFATc1 $(p<0.05)$ in all groups, except in Groups 1 and 4, and significant differences for the apoptotic index $(p<0.05)$ in all groups.

Conclusion Curcumin proved to be potentially effective in the prevention and treatment for fibroblasts damage within the cochlear supporting tissues and lateral wall regarding the decreased expression of calcineurin, NFATc1, and apoptotic index of cochlear fibroblasts.
\end{abstract}

\section{Introduction}

Exposure to excessive noise is the major avoidable cause of permanent hearing impairment. ${ }^{1}$ It is mostly found in the developing and industrial countries with bad hearing conservation. ${ }^{2}$ In 2012, there were 360 million persons in the world with disabling hearing loss, $\sim 5.3 \%$ of the world's population, of which 328 million (91\%) were adults (183 million men, 145 million women) and 32 (9\%) million were children. Sixteen per cent of the disabling hearing loss in the adult population in the world resulted from excessive noise exposure in the workplace, ranging from $7 \%$ to $21 \%$ in the various subregions. ${ }^{1,3}$ received

October 3, 2015

accepted

December 11, 2015

published online

March 4, 2016
DOI http://dx.doi.org/

10.1055/s-0036-1579742. ISSN $1809-9777$.
Copyright $(2016$ by Thieme Publicações License terms

Ltda, Rio de Janeiro, Brazil
(ब) $\Theta \circledast$ 
The cochlear spiral ligament is a connective tissue lining the space between stria vascularis and the bony otic capsule. It plays diverse roles in normal hearing and is composed of sub-populations of specialized fibrocytes, which are suggested to play distinct roles in fluid homeostasis, inflammatory responses, predicted by their protein expression profiles. Certain fibrocyte sub-types express ion transport proteins and, thus, are likely to regulate $\mathrm{K}^{+}$and $\mathrm{Cl}^{-}$within the lateral wall perilymph. ${ }^{4}$

Evidence from various cell lines shows that cell death can be stimulated by oxidative stress and excitotoxicity through $\mathrm{Ca}^{2+}$ overload. Acoustic overstimulation increases the $\mathrm{Ca}^{2+}$ concentration in auditory hair cells. Elevated $\mathrm{Ca}^{2+}$ has been implicated in the impairment of hair cell function and may initiate hair cell damage after noise exposure. There are several pathways through which $\mathrm{Ca}^{2+}$ may contribute to cell death, involving activation of nitric oxide synthase (NOS), phospholipase A2, proteases, and calcineurin. ${ }^{5}$

For years, studies have emerged based on the use of natural compounds plant-derived as potential therapeutic agents for various diseases in humans. ${ }^{6}$ Curcumin, a yellow pigment obtained from the rhizomes of Curcuma longa Linnaeus (Family: Zingiberaceae), is a major component of turmeric and has been used as a traditional medicine that possesses therapeutic potential against various diseases. Curcumin is capable of modulating numerous molecular targets involved in each stage of disease development by regulating transcription factors, growth factors, receptors, cytokines, kinases, enzymes, cell survival, metastatic, and apoptotic molecules. ${ }^{7}$

The role of curcumin in the prevention of and treatment of fibroblast damage within the supporting tissues and the cochlear lateral wall through the apoptosis inhibition mechanism contributed by calcineurin in cochlear fibroblasts has never been studied and serves as the focus in this study. The objective of this study is also to demonstrate that higher doses of curcumin ( $100 \mathrm{mg} /$ day) exert more beneficial effects in inhibiting apoptosis rather than low doses of curcumin (50 mg/day).

\section{Methods}

This study is an experimental study with randomized posttest-only control group design using male Wistar strain white Rattus norvegicus rats (150 - 250 g, 8 - 12 weeks of age). The dose and frequency of noise exposure was $100 \mathrm{~dB}$ SPL and $1-10 \mathrm{kHz}$ for 2 hours.

Curcumin used in this study was derived from Curcuma longa Linnaeus (Turmeric) with curcumin content levels of $28.1 \pm 1.0 \% \mathrm{w} / \mathrm{w}$ compared with standard, suspended in $0.5 \%$ carboxymethyl cellulose. Afterwards, we administered the suspension directly to the stomach of each rat via nasogastric tube, once a day for two weeks. The samples were composed of 24 Rattus norvegicus divided into 4 groups. Group 1: the control group; Group 2: noise (+); Group 3: noise (+), $50 \mathrm{mg}$ / day curcumin $(+)$; Group 4: noise $(+), 100 \mathrm{mg} /$ day curcumin $(+)$. We provided noise exposure doses of $100 \mathrm{~dB}$ SPL for two hours over two weeks.
After two weeks, the rats underwent termination by ether inhalation and necropsy procedure on their temporal bone. All samples underwent standard tissue processing with fixation in buffered formaldehyde, followed by dehydration in graded alcohol solutions. Thereafter, they were embedded in paraffin blocks, serially cut into $4 \mu \mathrm{m}$ thick sections, and put on glass slides. Representative sections were stained with hematoxylin and eosin ( $\mathrm{H} \& \mathrm{E})$. We performed immunohistochemical staining to examine the expressions of calcineurin and nuclear factor of activated T-cells cytoplasmic 1 (NFATc1) and apoptotic index of cochlear fibroblasts by terminal deoxynucleotidyl transferase (TdT) 2'-deoxyuridine 5'-triphosphate (dUTP) nick-end labeling (TUNEL) Assay.

Immunohistochemistry procedures were performed as follows. We cleared the slide in xylene and rehydrated it through graded series of alcohol solutions. Endogenous peroxidase activity was blocked with $3 \%$ hydrogen peroxide in absolute methanol. We prevented nonspecific binding of the second layer antibody by incubation with $10 \%$ nonimmune serum $(0.25 \%$ Triton X-100 in phosphate-buffered saline phosphate-buffered saline). Anti-Calcineurin A antibody (abcam ab71149, Abcam plc., Cambridge, USA) and NFATc1 antibody 7A6 (sc-7294, Santa Cruz Biotechnology, Inc., Dallas, Texas, USA) served as the first antibodies and were separately applied to each specimen and incubated in a humid chamber. After rinsing with phosphate-buffered saline, we incubated sections with biotinylated secondary antibody. Later, we washed them once more and incubated with a horseradish streptavidin-peroxidase conjugate. Next, we added a substrate-chromogen solution (3-3'-diaminobenzidine tetrahydrochloride). This reaction involved peroxidase catalysis of the substrate and conversion of the chromogen to a brown deposit that marked the antigen. The final steps included counterstaining with $\mathrm{H} \& \mathrm{E}$ and application of coverslips.

The TUNEL assay (The ApopTag Plus Peroxidase In Situ Apoptosis Detection Kit (Merck Millipore Corporation, Darmstadt, Germany) procedures were described as follows. We cleared the slide in xylene and rehydrated by transferring the slides through a graded ethanol series. We blotted away the excess water carefully and added proteinase K solution to cover sections. Afterwards, it was incubated at room temperature. We inactivated endogenous peroxidases by covering sections with $2 \%$ hydrogen peroxide. Later, the slide was washed and the excess water also was blotted away carefully. We added TdT equilibration buffer to cover sections and then removed the buffer. TdT reaction buffer was added to cover sections afterwards. The slide was incubated in a humidified chamber. To conserve reagents, a reduced volume of TdT buffer may be carefully covered with a glass coverslip during the incubation. The reaction was stopped by incubating the slide. Then we rinsed it in phosphate-buffered saline and blocked nonspecific binding by covering tissue sections with $2 \%$ Bovine Serum Albumin solution. Later, the slide was incubated in working strength stop/wash buffer. After applying stop solution, the sample was washed and incubated with anti-digoxigenin peroxidase conjugate. We developed the slide with 3-3'-diaminobenzidine tetrahydrochloride substrate, counterstained with methyl green, dehydrated, and cover-slipped. 


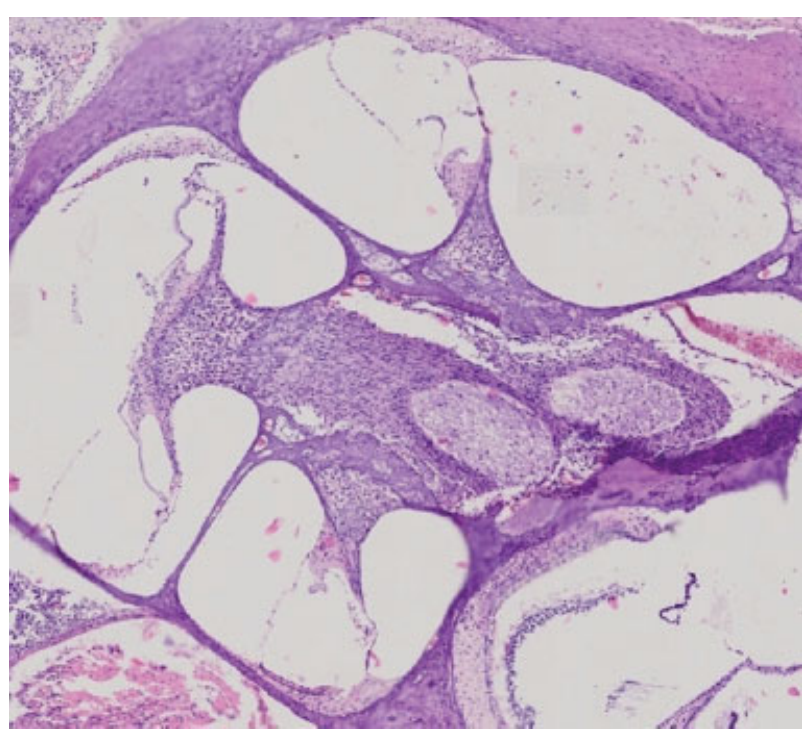

Fig. 1 The cochlear supporting tissues and lateral wall with $\mathrm{H} \& \mathrm{E}$ staining (20x zoom).

Three observers examined the samples in each slide. The fibroblasts within the cochlear supporting tissues and lateral wall, which expressed calcineurin and NFATc1, and apoptotic index in all fields were calculated manually with a hand counter. We calculated the expressions of calcineurin and NFATc1 quantitatively for the average distribution of fibroblasts unit with single nucleus expressing calcineurin and NFATc1 (showing brown-colored cytoplasms) and the occurrence of apoptosis (showing brown-colored nucleus).

We processed the data using the Statistical Package for the Social Sciences (SPSS) one-way analysis of variance (ANOVA) and used a $p$ value of 0.05 as the cut-off for statistical significance.

\section{Results}

We performed H\&E staining of rat cochlea to get a detailed view of the tissue (-Fig. 1 ).

The expression of calcineurin, after being evaluated with the immunohistochemistry method, showed an increased expression in Group 2 (-Fig. 2B), compared with other groups. The curcumin-treated groups showed lower density seen in the brown color, and less calcineurin-expressed fibroblasts than Group 2 (-Fig. 2C, D).

Data in -Table 1 show significant differences for the expressions of calcineurin $(p<0.05)$ in all groups. A dose of curcumin $100 \mathrm{mg}$ per day showed statistically significant decreases in the expressions of calcineurin rather than a dose of curcumin at $50 \mathrm{mg}$ per day.

The expression of NFATc1 after being evaluated with the immunohistochemistry method showed an increased expression in Group 2 (-Fig. 3B) compared with other groups. The curcumin-treated groups showed less NFATc1expressed fibroblasts than Group 2 (-Fig. 3C, D).

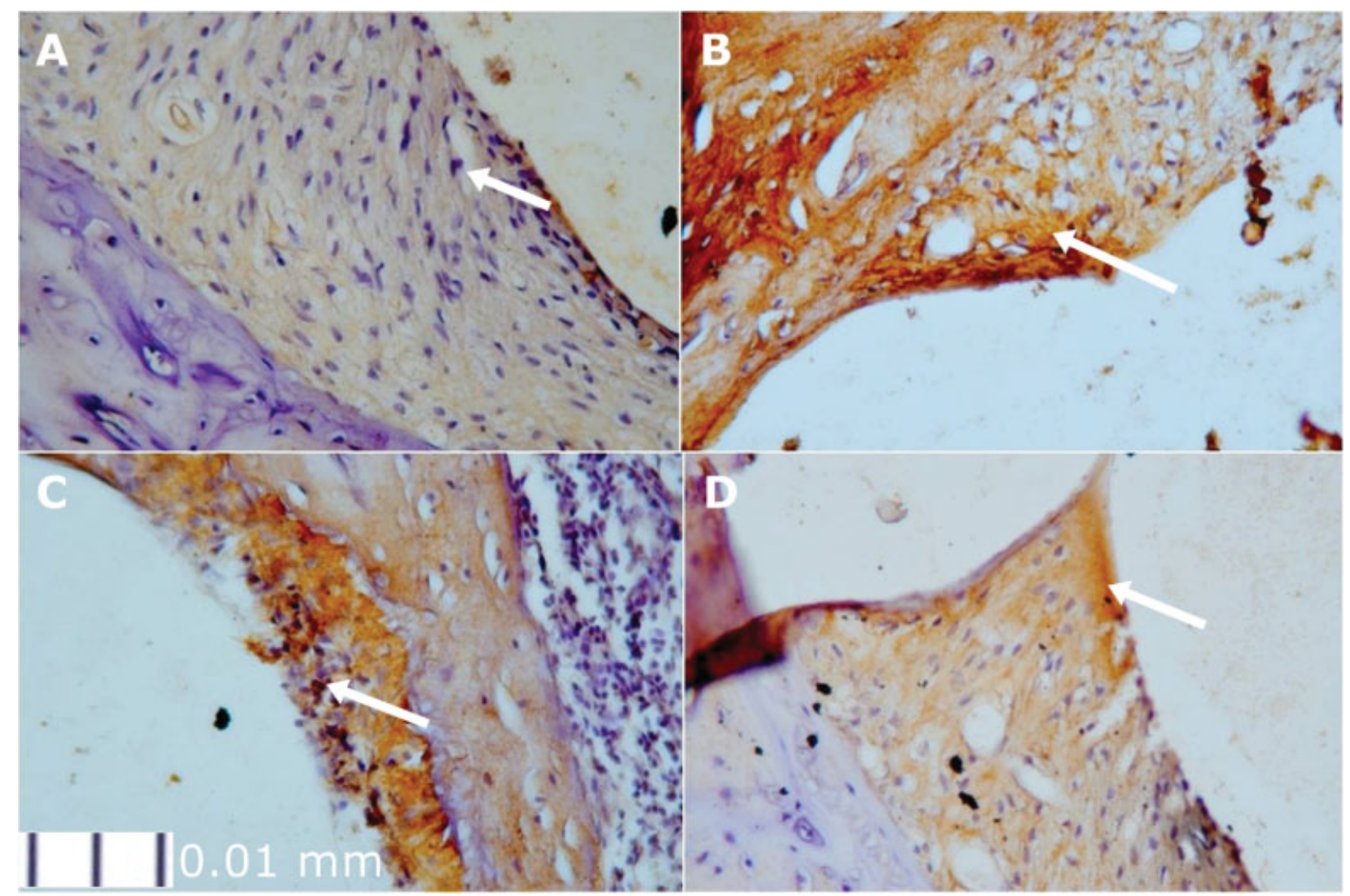

Fig. 2 The expression of calcineurin in each group (1000x zoom): (A) Group 1/control; (B) Group 2; (C) Group 3; (D) Group 4. The white arrow indicates the expression of calcineurin in cochlear fibroblasts marked by the brown color. 
Table 1 ANOVA test results in terms of the expressions of calcineurin

\begin{tabular}{|l|l|l|l|}
\hline \multicolumn{2}{|l|}{ Group } & $\begin{array}{l}\text { Mean difference } \pm \\
\text { Standard deviation }\end{array}$ & $P$ value \\
\hline Group 1 & Group 2 & $22.000 \pm 1.392$ & $0.000^{*}$ \\
\hline & Group 3 & $17.500 \pm 1.392$ & $0.000^{*}$ \\
\hline & Group 4 & $12.500 \pm 1.392$ & $0.000^{*}$ \\
\hline Group 2 & Group 3 & $4.500 \pm 1.392$ & $0.025^{*}$ \\
\hline & Group 4 & $9.500 \pm 1.392$ & $0.000^{*}$ \\
\hline Group 3 & Group 4 & $5.000 \pm 1.392$ & $0.011^{*}$ \\
\hline
\end{tabular}

*Denotes statistically significant.

Data in - Table 2 above showed significant differences for the expressions of NFATc1 $(p<0.05)$ in all groups, except in Groups 1 and 4 . A dose of curcumin of $100 \mathrm{mg}$ per day showed statistically significant decreases in the expressions of NFATc1, rather than a dose of curcumin $50 \mathrm{mg}$ per day.

The apoptotic index, after being evaluated with TUNEL assay showed an increased apoptotic index in group 2 (-Fig. 4 B) compared with other groups. The curcumintreated groups showed less apoptotic cells-expressed fibroblasts than group 2 (-Fig. 4C, D).
Data in -Table 3 showed significant differences for the apoptotic index $(p<0.05)$ in all groups. A dose of curcumin $100 \mathrm{mg}$ per day showed statistically significant decreases in the apoptotic index rather than a dose of curcumin $50 \mathrm{mg}$ per day.

\section{Discussion}

Acoustic overstimulation induces $\mathrm{Ca}^{2+}$ overload and mediated cell death pathways, involving activation of calcineurin. ${ }^{5,8}$ In this study, we found that the expression of calcineurin statistically increases in the cochlear fibroblast noiseexposed group (Group 2) when compared with the control group. Calcineurin belongs to the family of $\mathrm{Ca}^{2+} /$ calmodulindependent protein phosphatases, protein phosphatase $2 \mathrm{~B}$. Calcineurin is activated by binding of $\mathrm{Ca}^{2+} /$ calmodulin and the only protein phosphatase regulated by a second messenger $\mathrm{Ca}^{2+} \cdot 9,10$

It has been recently reported that calcineurin is activated in outer hair cells following noise exposure in mice exposed to broadband noise $(2-20 \mathrm{kHz}) .{ }^{8}$ In another experimental study in guinea pigs, after intense noise exposure $(4-10 \mathrm{kHz}$, $120 \mathrm{~dB}$, for 5 hours), varying degrees of hair cells loss and calcineurin immunoreactivity were detected immunohistochemistrically in outer hair cells and concentrated at the cuticular plate. $^{5}$

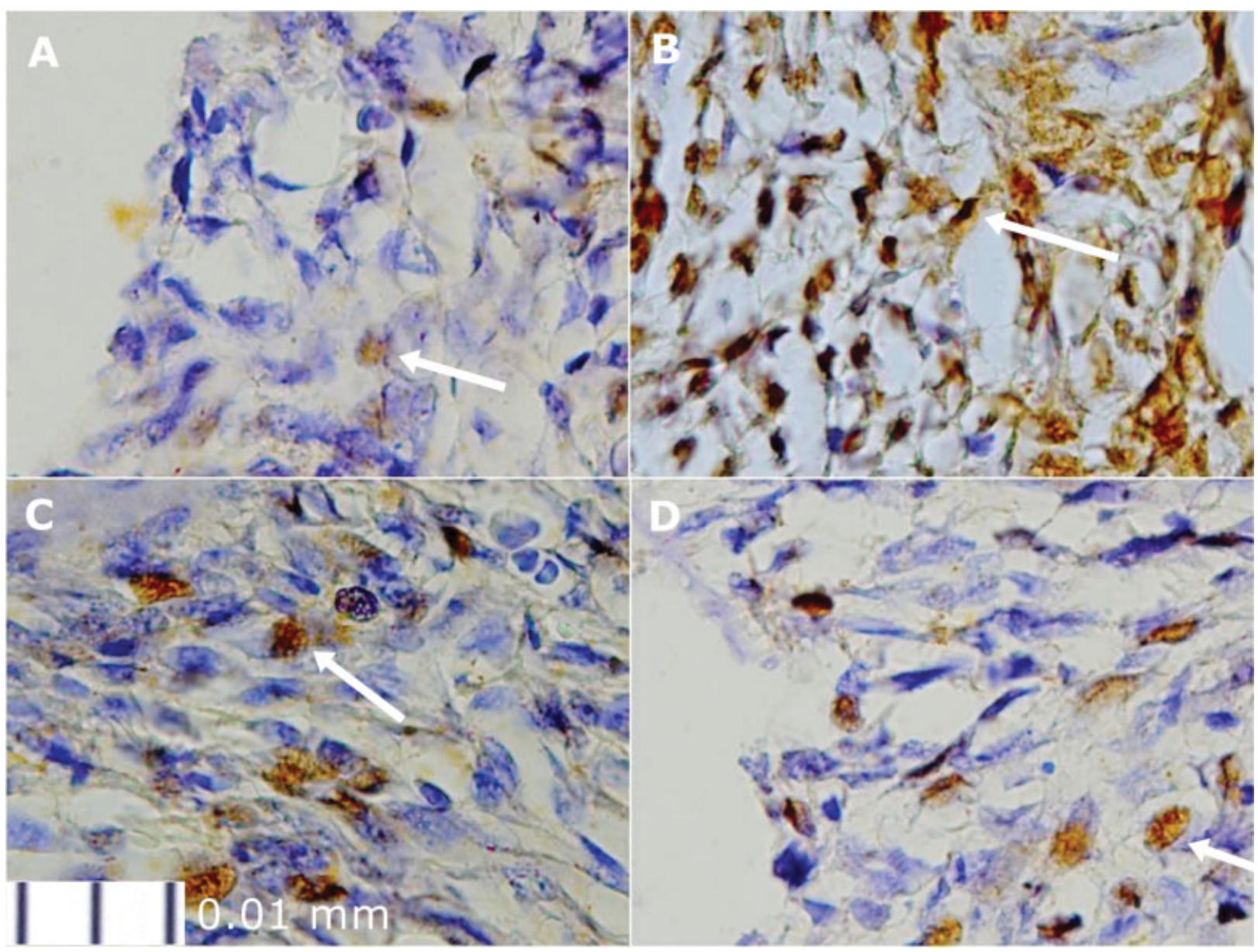

Fig. 3 The expression of NFATc1 in each group (1000x zoom): (A) Group 1/control; (B) Group 2; (C) Group 3; (D) Group 4. The white arrow indicates the expression of NFATc1 in cochlear fibroblasts marked by the brown color. 
Table 2 ANOVA test results in terms of expressions of NFATc1

\begin{tabular}{|l|l|l|l|}
\hline \multicolumn{2}{|l|}{ Group } & $\begin{array}{l}\text { Mean difference } \pm \\
\text { Standard deviation }\end{array}$ & $P$ value \\
\hline Group 1 & Group 2 & $10.833 \pm 0.792$ & $0.000^{*}$ \\
\hline & Group 3 & $5.667 \pm 0.792$ & $0.000^{*}$ \\
\hline & Group 4 & $1.833 \pm 0.792$ & 0.189 \\
\hline Group 2 & Group 3 & $5.167 \pm 0.792$ & $0.000^{*}$ \\
\hline & Group 4 & $9.000 \pm 0.792$ & $0.000^{*}$ \\
\hline Group 3 & Group 4 & $3.833 \pm 0.792$ & $0.001^{*}$ \\
\hline
\end{tabular}

*Denotes statistically significant.

The expression of NFATc1 was found to be statistically higher in the cochlear fibroblast noise-exposed group (Group 2 ) compared with the control group. $\mathrm{Ca}^{2+}$ overload activates calcineurin-dephosphorylated NFATs, leading to their translocation to the nucleus. In addition to this first wave of NFAT activation, in a second step of NFATc1/ $\alpha$ A generation, a short isoform of NFATc1 is strongly induced. ${ }^{11,12}$

The apoptotic cochlear fibroblasts were statistically higher in the cochlear fibroblast noise-exposed group (Group 2) compared with the control group. Calcineurin has a dual function and may exert its effects on apoptosis either by the
Table 3 ANOVA test results in terms of the apoptotic index

\begin{tabular}{|l|l|l|l|}
\hline \multicolumn{2}{|l|}{ Group } & $\begin{array}{l}\text { Mean difference } \pm \\
\text { Standard deviation }\end{array}$ & $P$ value \\
\hline Group 1 & Group 2 & $12.333 \pm 1.004$ & $0.000^{*}$ \\
\hline & Group 3 & $6.833 \pm 1.004$ & $0.000^{*}$ \\
\hline & Group 4 & $3.667 \pm 1.004$ & $0.010^{*}$ \\
\hline Group 2 & Group 3 & $5.500 \pm 1.004$ & $0.000^{*}$ \\
\hline & Group 4 & $8.667 \pm 1.004$ & $0.000^{*}$ \\
\hline Group 3 & Group 4 & $3.167 \pm 1.004$ & $0.030^{*}$ \\
\hline
\end{tabular}

*Denotes statistically significant.

activation of specific transcriptional pathways or by direct dephosphorylation of proteins including Bad (B cell lymphoma 2 antagonist of cell death) and caspase- 9 involved in the apoptotic pathway. ${ }^{10,11}$

Calcineurin dephosphorylates Bad, resulting in the disruption of the binding of Bax (B cell lymphoma 2 associated $\mathrm{x}$ protein) to $\mathrm{Bcl}-2$ (B cell lymphoma 2 ) or $\mathrm{Bcl}-\mathrm{xL}$ (B cell lymphoma 2-extra large) at the outer membrane of mitochondria through intrinsic pathway. Free Bax translocate to mitochondria and activate the transport system to release cytochrome c. $^{8,13,14}$

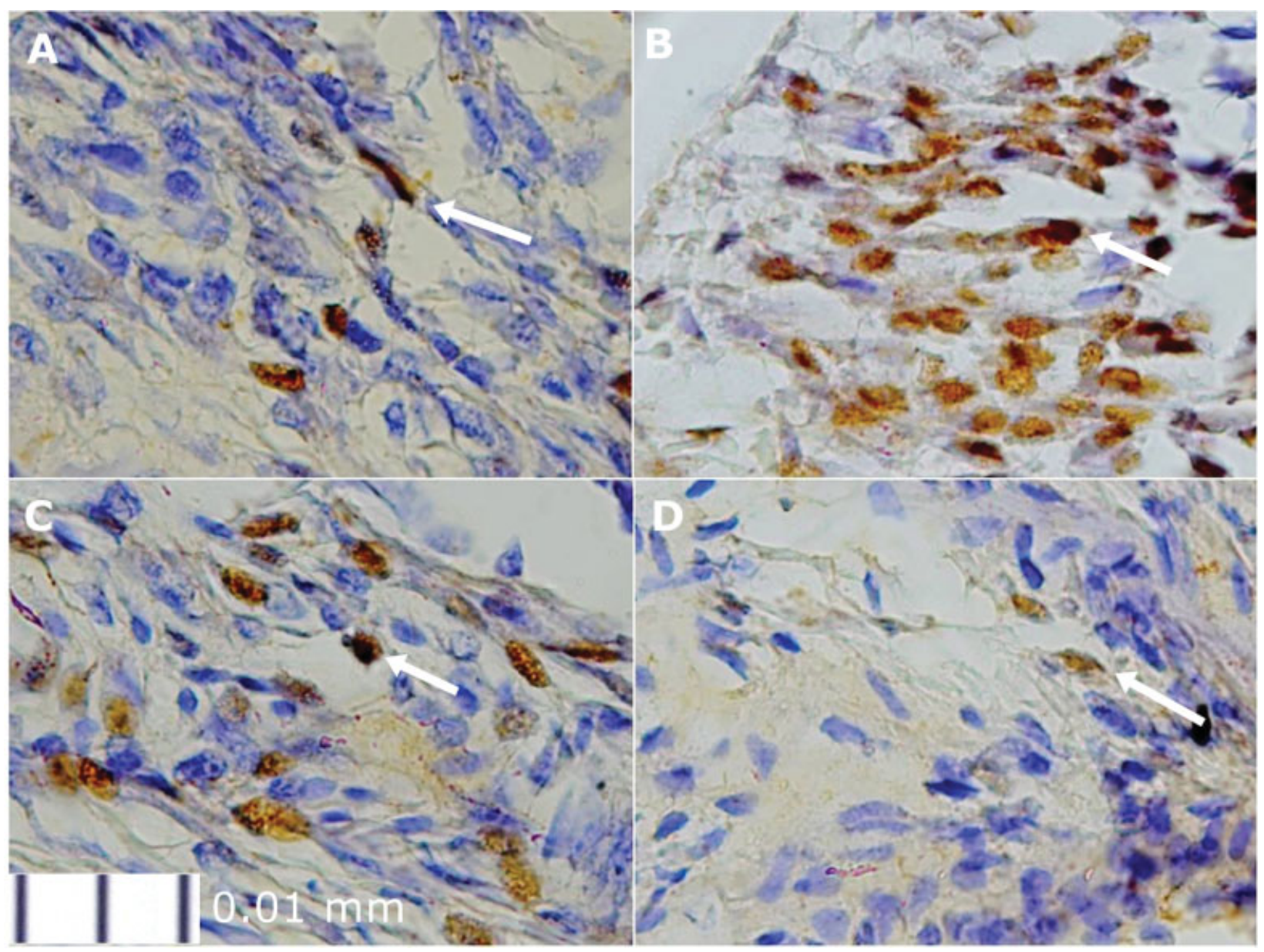

Fig. 4 The apoptotic index in each group (1000x zoom): (A) Group 1/control; (B) Group 2; (C) Group 3; (D) Group 4. The white arrow indicates the apoptotic cochlear fibroblasts marked by the brown color. 
In the extrinsic pathway, outside the mitochondria, it has been previously described that expression of the membranebound death receptor ligand FasL is mediated by NFAT. When FasL binds to its receptor Fas, the intracellular machinery associated with the death receptor Fas is activated and eventually leads to apoptosis by caspase activation and subsequent DNA cleavage. ${ }^{2}$ Caspase- 8 directly cleaves caspase-3 as well as cytosolic Bid (BH-3 interacting domain death agonist), its active fragment (tBid) translocates to mitochondria to release cytochrome $\mathrm{c}^{15}$

Cytochrome $c$ interacts with proteins such as Apaf-1 (apoptotic protease activating factor-1), dATP, and procaspase-9 to produce apoptosome and then activates caspase9. This complex degrades procaspase- 3 to caspase $-3 .{ }^{15}$ In apoptotic cells, activated caspase- 3 cleaves inhibitor of caspase activated DNAse (ICAD) to release CAD. CAD then degrades chromosomal DNA within the nuclei and causes chromatin condensation. Caspase-3 also induces cytoskeletal reorganization and disintegration of the cell into apoptotic bodies. $^{14}$

An experimental study in mice exposed to broadband noise $(2-20 \mathrm{kHz}$ ) has discovered that noise exposure induces activation of mitochondria-mediated cell death pathways in outer hair cells of the cochlea through activation of Bad by calcineurin. The localization of Bad was analyzed by immunohistochemistry. Total Bad was observed both in the sensory cells and in the supporting cells and also in the nerve fibers projecting to the sensory cells. The results of this study strongly support the Bad as a link between $\mathrm{Ca}^{2+}$ influx after noise exposure and the death of outer hair cells. ${ }^{8}$ In another study, after intense noise exposure ( 4 - $10 \mathrm{kHz}, 120 \mathrm{~dB}$, for 5 hours) in guinea pigs, some calcineurin-immunopositive hair cells demonstrated condensed and swollen nuclei, indicating that calcineurin is related to both apoptosis and necrosis. ${ }^{5}$

This study proved that curcumin was able to decrease the expression of calcineurin and NFATc1 in cochlear fibroblasts, where a dose of curcumin $100 \mathrm{mg}$ per day showed statistically significant decreases in the expression of calcineurin and NFATc1 compared with a dose of curcumin $50 \mathrm{mg}$ per day. This is due to the speculation that curcumin inhibits the regulation and expression of calcineurin, and prevents the dephosphorylation of NFATc1 by calcineurin, thus, reducing its translocation to the nucleus. The expression of NFATc1 in Group 4 was statistically insignificant compared with the control group, indicating that curcumin administration at higher doses is able to prevent NFATc1 activation, thereby its expression was found to be nearly similar to the control group (without noise exposure).

Researchers have recently investigated the therapeutic efficacy of curcumin in attenuation of left ventricular hypertrophy and sought to delineate the associated signaling pathways in blunting the hypertrophic response in nephrectomized rats. Curcumin attenuates cardiac hypertrophy and remodeling through deactivation of multiple hypertrophic signaling pathways. This study reported that cytosolic NFAT was significantly decreased in rats that underwent nephrectomy and was significantly attenuated by curcumin. NFAT in the nucleus was decreased by curcumin with quantitative real-time reverse transcription-polymerase chain reaction (RT-PCR) analysis. ${ }^{16}$ Another study showed an action of curcumin as an NFAT inhibitor through the $\mathrm{Ca}^{2+}$ signaling pathway blocking. This experiment also demonstrates that curcumin inhibits NFAT transcriptional activity by preventing its nuclear translocation from the cytoplasm into the nucleus upon phorbol myristate acetate (PMA)/ionomycin stimulation of Jurkat T-cells with laser scan microscopy (LSM) analysis. ${ }^{17}$

Curcumin significantly promoted nonischemic wound healing in a dose-response fashion compared with controls as judged by increased reepithelialization and granulation tissue formation. Improved wound healing was associated with significant decreases in pro-inflammatory cytokines interleukin (IL)-1 and IL-6 as well as the chemokine IL-8. Curcumin also significantly reduced hypertrophic scarring. ${ }^{18}$

Another study concluded that pre- and coreceiving curcumin can significantly protect the cochlear morphology and functions on paclitaxel-induced ototoxicity in rats using light microscopy and distortion product otoacoustic emissions (DPOAEs) to evaluate histopathological, immunohistochemical, and functional changes in hearing. Curcumin might be considered as a potential dietary supplement from a natural product given to patients undergoing paclitaxel chemotherapy. ${ }^{19}$

Curcumin can also be used as an efficient adjuvant to cisplatin cancer therapy. This treatment strategy in head and neck cancer could mediate cisplatin chemoresistance by modulating therapeutic targets (Signal transducer and activator of transcription 3 and NF-E2 p45-related factor 2) and, at the same time, reduce cisplatin-related ototoxic adverse effects. $^{20}$

Preclinical studies demonstrated that systemic curcumin attenuates ototoxicity and provides molecular evidence for a role of hemeoxigenase (HO-1) as an additional mediator in attenuating cisplatin-induced hearing loss. ${ }^{21}$

A previous study found the effect of curcumin on peroxynitrite (ONOO)-induced damage in rat spiral ganglion neurons. Pretreatment with curcumin abrogated cytochrome c release, blocked activation of caspase-3, and altered the expression of Bcl-2 family triggered by ONOO. Curcumin can attenuate ONOO-induced damage in spiral ganglion neurons by the anti-oxidative activity, as well as protect mitochondria from oxidative stress. ${ }^{22}$

In the present study, we proved that curcumin is able to decrease the apoptotic cochlear fibroblasts, whereby a dose of curcumin $100 \mathrm{mg}$ per day showed significant decreases in the apoptotic index compared with a dose of curcumin $50 \mathrm{mg}$ per day. This is due to the speculation that curcumin inhibits calcineurin activation, therefore its role in Bad dephosphorylation can be prevented. Furthermore, the complex of Bcl-2 or $\mathrm{Bcl}-\mathrm{XL}$ remains inseparable and both function in preventing cytochrome $\mathrm{c}$ release into cytosol is inhibitable and apoptosis becomes an avoidable process. Curcumin also inhibits the dephosphorylation of NFATc1 leading to reduced translocation to the nucleus, therefore, preventing apoptosis by caspase- 8 activation. 


\section{Conclusion}

This study indicates that curcumin is safe and effective as a therapeutic agent in the prevention and treatment of the damage of fibroblasts within the supporting tissues and the cochlear lateral wall through the cell death pathway. Moreover, the study provides more insight into the mechanism of curcumin against apoptosis and shows that curcumin inhibits multiple apoptosis signaling pathways, including calcineurin and NFATc1. The study may serve as a scientific basis in the traditional systems of medicine for the management of NIHL in the future.

\section{References}

1 Nandi SS, Dhatrak SV. Occupational noise-induced hearing loss in India. Indian J Occup Environ Med 2008;12(2):53-56

2 Harmadji S, Kabullah H. Noise induced hearing loss in steel factory workers. Folia Medica Indonesiana 2004;40(4):171-174

3 World Health Organization. WHO global estimates on prevalence of hearing loss. Available at: http://www.who.int/pbd/deafness/ WHO_GE_HL.pdf. Accessed Dec 10, 2014

4 Kelly JJ, Forge A, Jagger DJ. Contractility in type III cochlear fibrocytes is dependent on non-muscle myosin II and intercellular gap junctional coupling. J Assoc Res Otolaryngol 2012;13(4):473-484

5 Minami SB, Yamashita D, Schacht J, Miller JM. Calcineurin activation contributes to noise-induced hearing loss. J Neurosci Res 2004;78(3):383-392

6 Trujillo J, Granados-Castro LF, Zazueta C, Andérica-Romero AC, Chirino YI, Pedraza-Chaverrí J. Mitochondria as a target in the therapeutic properties of curcumin. Arch Pharm (Weinheim) 2014;347(12):873-884

7 Prasad S, Gupta SC, Tyagi AK, Aggarwal BB. Curcumin, a component of golden spice: from bedside to bench and back. Biotechnol Adv 2014;32(6):1053-1064

8 Vicente-Torres MA, Schacht J. A BAD link to mitochondrial cell death in the cochlea of mice with noise-induced hearing loss. J Neurosci Res 2006;83(8):1564-1572

9 Morioka M, Hamada J, Ushio Y, Miyamoto E. Potential role of calcineurin for brain ischemia and traumatic injury. Prog Neurobiol 1999;58(1):1-30
10 Zhu H, Gao W, Jiang H, Wu J, Shi YF, Zhang XJ. Calcineurin mediates acetylcholinesterase expression during calcium ionophore A23187-induced HeLa cell apoptosis. Biochim Biophys Acta 2007;1773(4):593-602

11 Alvarez S, Blanco A, Fresno M, Muñoz-Fernández MA. TNF- $\alpha$ contributes to caspase-3 independent apoptosis in neuroblastoma cells: role of NFAT. PLoS ONE 2011;6(1):e16100

12 Serfling E, Avots A, Klein-Hessling S, Rudolf R, Vaeth M, BerberichSiebelt F. NFATc $1 / \alpha A$ : The other Face of NFAT Factors in Lymphocytes. Cell Commun Signal 2012;10(1):16

13 Precht TA, Phelps RA, Linseman DA, et al. The permeability transition pore triggers Bax translocation to mitochondria during neuronal apoptosis. Cell Death Differ 2005;12(3):255-265

14 Elmore S. Apoptosis: a review of programmed cell death. Toxicol Pathol 2007;35(4):495-516

15 Maher S, Toomey D, Condron C, Bouchier-Hayes D. Activationinduced cell death: the controversial role of Fas and Fas ligand in immune privilege and tumour counterattack. Immunol Cell Biol 2002;80(2):131-137

16 Ghosh SS, Salloum FN, Abbate A, et al. Curcumin prevents cardiac remodeling secondary to chronic renal failure through deactivation of hypertrophic signaling in rats. Am J Physiol Heart Circ Physiol 2010;299(4):H975-H984

17 Kliem C, Merling A, Giaisi M, Kohler R, Krammer PH, Li-Weber M. Curcumin suppresses $\mathrm{T}$ cell activation by blocking $\mathrm{Ca} 2+$ mobilization and nuclear factor of activated T cells (NFAT) activation. J Biol Chem 2012;287(13):10200-10209

18 Jia S, Xie P, Hong SJ, et al. Intravenous curcumin efficacy on healing and scar formation in rabbit ear wounds under nonischemic, ischemic, and ischemia-reperfusion conditions. Wound Repair Regen 2015;22(6):730-739

19 Bucak A, Ozdemir C, Ulu S, et al. Investigation of protective role of curcumin against paclitaxel-induced inner ear damage in rats. Laryngoscope 2015;125(5):1175-1182

20 Fetoni AR, Paciello F, Mezzogori D, et al. Molecular targets for anticancer redox chemotherapy and cisplatin-induced ototoxicity: the role of curcumin on pSTAT3 and Nrf-2 signalling. Br J Cancer 2015;113(10):1434-1444

21 Fetoni AR, Eramo SLM, Paciello F, et al. Curcuma longa (curcumin) decreases in vivo cisplatin-induced ototoxicity through heme oxygenase-1 induction. Otol Neurotol 2014;35(5):e169-e177

22 Liu W, Fan Z, Han Y, et al. Curcumin attenuates peroxynitriteinduced neurotoxicity in spiral ganglion neurons. Neurotoxicology 2011;32(1):150-157 